\title{
Semileptonic Decays of Heavy Mesons: A Status Report*
}

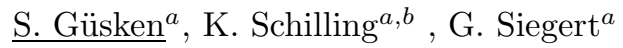 \\ ${ }^{a}$ Physics Department, University of Wuppertal, D-42097 Wuppertal, Germany \\ ${ }^{b}$ HRLZ, c/o KFA, D-52425 Jülich, Germany
}

\begin{abstract}
We present intermediate results on our ongoing investigation concerning semileptonic decays of heavy pseudoscalar mesons into pseudoscalar and vector mesons. The corresponding formfactors are evaluated at several momenta and appropriate combinations of four light and four heavy quarks, which are chosen to allow for an extrapolation into the B Meson region. In order to obtain clear groundstate signals we apply gauge invariant "Wuppertal" smearing to the quarks. The analysis is based on 32 quenched gauge configurations of size $24^{3} \times 64$ at $\beta=6.3$, with Wilson fermions.
\end{abstract}

\section{INTRODUCTION}

The accurate determination of Kobayashi Maskawa (KM) matrix elements involving beauty quarks is an important but also very challenging task to both experimental and theoretical physics. On the theoretical side nonpertubative methods are indispensable to calculate the low energy QCD parts of the transition amplitudes. Although lattice QCD is the method of choice in this context, it suffers from the fact that the B meson region is beyond currently reachable lattice resolutions. Direct lattice calculations at the B meson mass would be contaminated by large discretization errors.

In the current project we are aiming at a high statistics determination, with about 100 independent configurations, of the relevant weak formfactors, at a lattice resolution of $a^{-1} \simeq 3.2 \mathrm{GeV}$. This resolution allows to push forward into a mass region of $(1-1.5) \times m_{D}$, with tolerable discretization errors. We are running at four values of the heavy quark mass $m_{h}$, with hopping parameters $\kappa_{h}=0.1200,01300,01350,0.1400$ and four values of the light quark mass $m_{l}, \kappa_{l}=$ $0.1450,0.1490,0.1507,0.1511$. This covers the physical regions $0.8 m_{c} \leq m_{h} \leq 1.6 m_{c}$ and $0.8 m_{s} \leq m_{l} \leq 3 m_{s}$ respectively, providing us with sufficient lever arms for the extrapolations to $m_{u}$ and $m_{b}$.

\footnotetext{
*Work supported by DFG grant Schi 257/1-4, Schi 257/32, EC contract CHRX-CT92-0051 and Mu 810/3.
}

In this status report, we present the results of an intermediate analysis, based on 32 configurations.

\section{LATTICE SIGNALS}

We consider the formfactors parametrizing the decay of pseudoscalar mesons, $P S \rightarrow$ $P S^{\prime}, f^{+}\left(q^{2}\right), \quad f^{0}\left(q^{2}\right)$, and the corresponding decays into vector mesons, $P S \rightarrow V$, $V\left(q^{2}\right), A_{1}\left(q^{2}\right), A_{2}\left(q^{2}\right), A\left(q^{2}\right)$. They can be extracted from the groundstate properties of appropriate ratios $R(t)$ of two- and three-point correlation functions[1]:2]. The three-point correlators are calculated using the well known insertion technique[3].

In our setup the initial meson is located at timeslice $t_{i}=32$ and carries no momentum, whereas the final meson is placed at timeslice $t_{f}=1$ and is furnished with altogether 11 lattice momenta, i.e. all combinations possible in the range $0 \leq\left|\vec{p}_{f}\right| \leq 2 p_{\text {min }}, p_{\text {min }}=2 \pi / 24 a$. The location in time of the effective elektroweak interaction is varied between $t_{i}$ and $t_{f}$ and must be chosen finally in the range of groundstate dominance, as signalled by the existence of a plateau.

In order to enhance groundstate signals we have applied Wuppertal "Gaussian" smearing泪 to all boundstate quarks, with an average radius of $\sqrt{\left\langle r^{2}\right\rangle} \simeq 5 a$. We obtain clear plateaus for all relevant ratios $R(t)$ with momenta $0 \leq\left|p_{f}\right| \leq$ $\sqrt{2} \times p_{\min }$ in the range $10 \leq t \leq 20$. For higher 
momenta the signals start getting noisy and a safe identification of the plateau region becomes increasingly difficult.

\section{3. $q^{2}$ Dependence}

In order to extract the $\mathrm{KM}$ matrix elements from experimental measurements of decay widths and branching ratios one conveniently uses the values of the formfactors at $q^{2}=0$. A natural guide for the extrapolation in $q^{2}$ is the pole dominance hypothesis

$F\left(q^{2}\right)=\frac{F(0)}{1-\frac{q^{2}}{m_{t}^{2}}}$.

$F$ denotes generically the formfactors given above and $m_{t}$ is the mass of the lightest meson exchanged in the $t$ channel. The ansatz can be checked by the "high momentum transfer" data, at $|\vec{p}|=\sqrt{3} p_{\min }$ and $2 p_{\min }$. In fig.11 we show
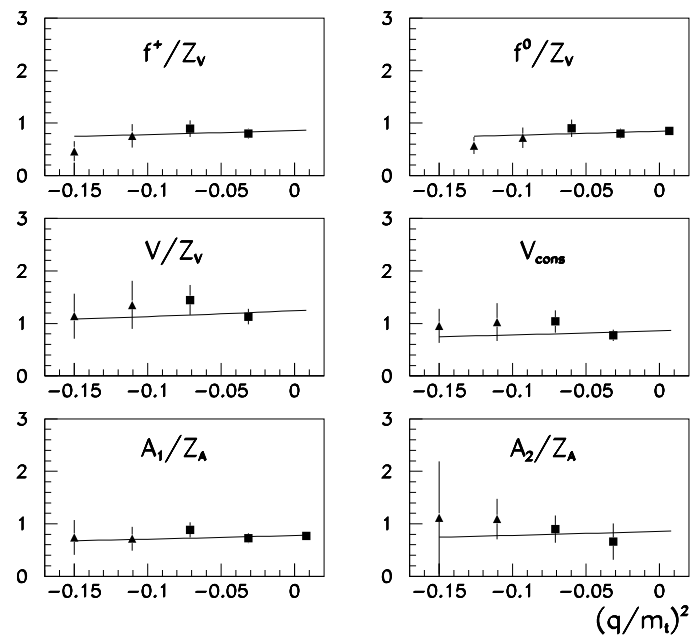

Figure 1. $\quad q^{2}$ dependence of formfactors. The mass of the heavy initial and the heavy final quark corresponds to $\kappa_{i}=0.120$ and $\kappa_{f}=0.135$ respectively. The mass of the light spectator quark is $\kappa_{\text {spec }}=0.1490$ and the mass $m_{t}$ was extracted from appropriate 2-point correlators. The triangles denote the "high momentum" results.

the $q^{2}$ dependence of the formfactors for a given combination of quark masses. The solid line corresponds to a fit to eq.11, where we have included only the most reliable low momentum results. We find reasonable consistency of all data with the pole dominance hypothesis. We would like to emphasize that the extrapolation to $q^{2}=0$ depends only weakly on the details of the ansatz as it is short ranged.

\section{D Meson Decays}

The charm quark region is directly accessed by our calculation. After extrapolation in $q^{2}$ and in the light quark mass $m_{l} \rightarrow m_{u}$ and $m_{l} \rightarrow m_{s}$ we find
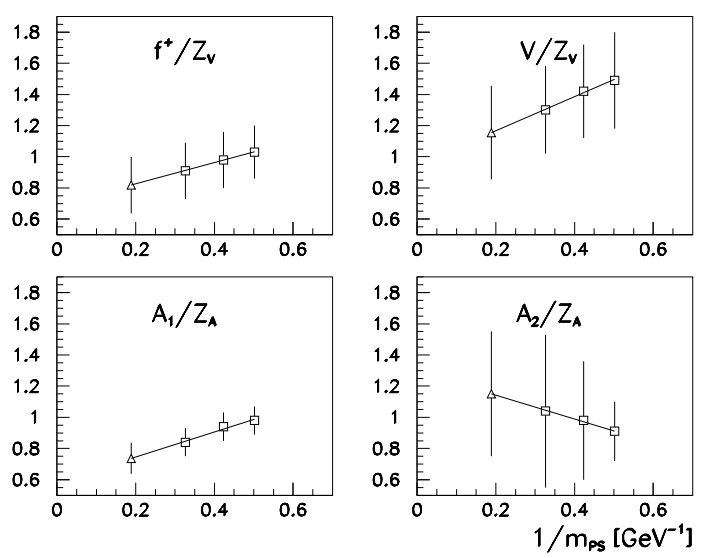

Figure 2. $1 / M_{p s}$ dependence of formfactors in the heavy quark regime. The light quark mass is extrapolated to the chiral point and the mass of the final heavy quark corresponds to $m_{c}$. The triangle represents the result of the fit at $M_{B}$.

$$
\begin{aligned}
& D \rightarrow K l \nu, K^{*} l \nu: \\
& f^{+}(0) / Z_{V}=1.02(20) \quad f^{0}(0) / Z_{V}=1.01(8) \\
& V(0) / Z_{V}=1.41(45) \\
& A_{1}(0) / Z_{A}=0.74(10) \quad A_{2}(0) / Z_{A}=0.70(47)
\end{aligned}
$$

and

$$
\begin{aligned}
& D \rightarrow \pi l \nu, \rho l \nu: \\
& f^{+}(0) / Z_{V}=1.07(22) \quad f^{0}(0) / Z_{V}=1.04(8) \\
& V(0) / Z_{V}=1.46(48) \\
& A_{1}(0) / Z_{A}=0.78(11) \quad A_{2}(0) / Z_{A}=0.73(52) .
\end{aligned}
$$

A discussion of the issue of the renormalization constants of the vector- and axial vector-currents, $Z_{V}$ and $Z_{A}$, will follow in section 6 . 


\section{5. $B$ Meson Decays}

The $B$ meson can be accessed only by extrapolation in the heavy quark mass. The heavy quark effective theory predicts[5]

$F\left(M_{p s}\right)=c+\frac{d}{M_{p s}}$,

if both the initial and the final quark are "heavy". In fig.2 we display our heavy quark results together with a fit to eq.2. Obviously the data, within the present statistics, is well described by eq.2, and we obtain for the transitions

$$
\begin{array}{ll}
B \rightarrow D l \nu, D^{*} l \nu: & \\
f^{+}(0) / Z_{V}=0.82(20) & V(0) / Z_{V}=1.15(30) \\
A_{1}(0) / Z_{A}=0.74(20) & A_{2}(0) / Z_{A}=1.15(60) .
\end{array}
$$

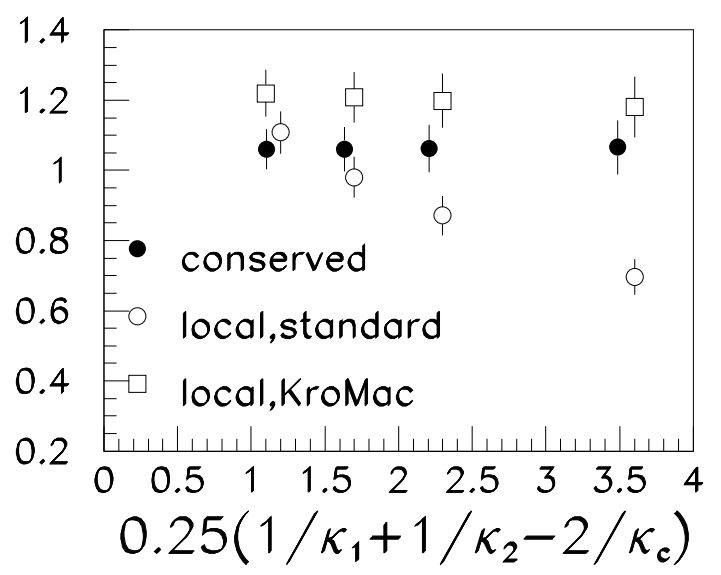

Figure 3. Mass dependence of $M_{l o c}$ and $M_{\text {cons }}$ for degenerate pseudoscalar states. The mass of the light spectator quark is $\kappa_{\text {spec }}=0.1490$.

\section{Mass Dependence of $Z_{V}$}

In order to convert the above lattice data into continuum results, we need the renormalization constants $Z_{V}$ and $Z_{A}$ of the vector and axialvector currents. They can be calculated in perturbation theory, but the estimation of the systematic error is difficult in this case.
Therefore it is important to gain information about the renormalization constants directly from the nonperturbative data.

One year ago C.W. Bernard [6] argued that the renormalization constant of the local vector current $Z_{V}$ depends strongly on the mass of the quarks involved. Following his arguments this undesired mass dependence can be removed if the (standard) $\sqrt{2 \kappa}$ normalization of the quark fields is replaced by the Kronfeld-Mackenzie(KroMac) (7) prescription.

As a first step to check this issue $2^{2}$ we display in fig. 3 the mass dependence of the matrix element $M_{l o c}=\left(\left\langle P S\left|V_{0}^{l o c}\right| P S\right\rangle / Z_{V}\right)_{q^{2}=0}$ with the standard and with the KroMac normalization. We have also included the result for the conserved current $M_{\text {cons }}=\left\langle P S\left|V_{0}^{\text {cons }}\right| P S\right\rangle_{q^{2}=0}$, which is expected to be 1 in the standard normalization because of the corresponding Ward identity.

Obviously the data favor the KroMac normalization for the local current, as for this choice $Z_{V}=M_{\text {cons }} / M_{l o c}$ becomes mass independent.

In a forthcoming paper we will extend the above considerations to the space components of the vector and axialvector currents.

Acknowledgements: We thank R. Gupta, A.S. Kronfeld and A. Vladikas for useful discussions related to section 6 .

\section{REFERENCES}

1. V. Lubicz et al.; Nucl. Phys. B356 (1991)301.

2. A. Abada et al.; Nucl. Phys. B416 (1994)675.

3. M. Crisafulli et al.; Phys. Lett. B223 (1989)90.

4. C. Alexandrou et al; Nucl. Phys. B414(1994)815.

5. N. Isgur and M.B. Wise; Phys. Rev. D42 (1990)2388.

6. C.W. Bernard; Nucl. Phys. B(Proc. Suppl.) 34(1994) 47.

7. A.S. Kronfeld, Nucl. Phys. B(Proc. Suppl.) 30(1993)445; P.B. Mackenzie, Nucl. Phys. B(Proc. Suppl.) 30(1993)35.

8. A. Vladikas et al.; these proceedings.

${ }^{2}$ see also 8 . 